\title{
EXEMPLES DE CLASSES D'AUTOMATES CELLULAIRES
}

\author{
Marianne Delorme $^{1,2}$ ET JACQUes MAZOYeR ${ }^{1,2}$
}

\begin{abstract}
Cellular automata classes: examples. Observing orbits of some cellular automata may lead to think that they are results of evolutions of other cellular automata, which could be considered as sort of components. In this paper, we try to understand this phenomenon by constructing a hybrid of two cellular automata by means of a third one. Two types of cellular automata are introduced: "captifs" and "foulards" cellular automata. We compare properties of hybrids in the framework of algebraic classifications introduced in [B. Martin (2001); N. Ollinger (2002); I. Rapaport (1998); G. Teyssier (2005): PhD. Thesis, École Normale Supérieure de Lyon].
\end{abstract}

Résumé. Lorsqu'on observe des orbites de certains automates cellulaires, on peut penser qu'elles apparaissent comme des mélanges d'orbites d'autres automates (composants). Dans cet article, nous tentons de comprendre ce phénomène en construisant un hybride de deux automates au moyen d'un troisième. Deux types d'automates cellulaires sont introduits : les captifs et les foulards. Nous comparons des propriétés de ces hybrides dans le cadre des classifications algébriques introduites par [B. Martin (2001) ; N. Ollinger (2002) ; I. Rapaport (1998) ; G. Teyssier (2005) : PhD. Thesis, École Normale Supérieure de Lyon].

Classification Mathématique. 68Q80, 37B15.

\section{INTRODUCTION}

Les recherches sur la dynamique des automates cellulaires se mènent actuellement suivant deux perspectives principales : soit celle de la connaissance détaillée d'un automate particulier, soit celle de l'étude d'une classe d'automates à l'aide

\footnotetext{
Mots Clés. Automates cellulaires, classification, auto-organisation, émergence.

Cellular automata, classification, self-organization, emergence.

1 Laboratoire de l'Informatique du Parallélisme, École Normale Supérieure de Lyon, 46 allée d'Italie, 69634 Lyon, France; mazoyer@ens-lyon.fr

2 Institut des Systèmes Complexes, IXXI, 5 rue du Vercors, Lyon 69007, France.

(c) EDP Sciences 2007
} 
d'outils provenant de la théorie des systèmes dynamiques continus utilisés dans un cadre discret et de la dynamique symbolique. On peut ranger dans cette seconde catégorie les travaux fondés sur des propriétés topologiques ou métriques, ou sur des extensions de ces propriétés à des pseudo-métriques par introduction de mesures [2,21,27]. Mais on connait fort peu de classes d'automates présentant une structure algébrique qui permette d'en faire une étude approfondie. Pour l'essentiel il s'agit des automates linéaires [28] dont la dynamique est complètement connue grâce aux polynômes qui leur sont canoniquement associés. On peut aussi y ranger les automates permutifs ${ }^{1}$ ou bi-permutifs $[25,38]$. En outre on ne dispose que de peu d'exemples d'automates intéressants hormis les 256 automates à deux états (dits élémentaires et désignés suivant la numérotation introduite par Wolfram [47]). Parmi ceux-ci, certains sont triviaux et bien compris (identité, décalage, les linéaires, les nilpotents), d'autres sont beaucoup plus compliqués, très étudiés et partiellement compris (comme les règles 184, 54 ou 110 par exemple) et semblent intuitivement indécomposables.

Nous nous intéressons ici à la problématique de composer des automates cellulaires de telle sorte que le composé ait des propriétés fortes qui se déduisent de celles de ses composantes. Nous cherchons donc à construire et à manipuler algébriquement de nouveaux exemples non triviaux, éventuellement à distinguer de nouvelles classes significatives. Et cela en évitant les explosions du voisinage ou du nombre d'états induites par la composition des fonctions (dans une vision globale des automates cellulaires suivant la caractérisation de Hedlund [16]) ou le produit cartésien, tout en obtenant une structure plus riche que la simple superposition. L'idée est alors de chercher à imposer des conditions sur la table de transition locale d'un automate, ce qui conduit par exemple à la notion d'automate cellulaire captif introduite par Theyssier [46], ou à amalgamer, suivant des conditions plus ou moins générales, des tables de transitions locales en une nouvelle table d'automate produit, ce qui conduit à des automates que nous avons baptisés foulards.

Nous nous placerons dans le cadre d'automates cellulaires de dimension 1 à voisinage $\{-1,0,1\}$. Ainsi un automate $\mathcal{A}$ est-il un couple $\mathcal{A}=\left(Q_{\mathcal{A}}, \delta_{\mathcal{A}}\right)$ où $Q_{\mathcal{A}}$ est un ensemble fini (les états de l'automate) et $\delta_{\mathcal{A}}$ est une application de $Q_{\mathcal{A}}^{3}$ dans $Q_{\mathcal{A}}$ (fonction locale de transition). A $\mathcal{A}=\left(Q_{\mathcal{A}}, \delta_{\mathcal{A}}\right)$ est associée la fonction $\Delta_{\mathcal{A}}$ de $Q_{\mathcal{A}}^{\mathbb{Z}}$ dans lui-même, dite fonction globale de $\mathcal{A}$. La suite $\left(\Delta_{\mathcal{A}}^{t}(c)\right)_{t \in \mathbb{N}}$ est l'orbite de $\mathcal{A}$ sur $c$; elle est représentée par l'empilement des configurations successives (le diagramme espace-temps de $\mathcal{A}$ sur $c$ ).

Définition 1. Un automate cellulaire $\mathcal{A}=\left(Q_{\mathcal{A}}, \delta_{\mathcal{A}}\right)$ sera dit captif [46] lorsque, pour tout $q_{1}, q_{2}, q_{3}$ de $Q_{\mathcal{A}}, \delta_{\mathcal{A}}\left(q_{1}, q_{2}, q_{3}\right) \in\left\{q_{1}, q_{2}, q_{3}\right\}$.

Cette notion d'automate captif reprend la notion de sous-alphabet invariant de [8], étudiée en [9-12]. La Figure $1^{2}$ montre une évolution assez typique de deux automates captifs à quatre états.

\footnotetext{
${ }^{1}$ Un automate $\mathcal{A}=(Q, \delta,\{-1,0,1\})$ sur $\mathbb{Z}$ est dit permutif à droite si quels que soient $q^{\prime}$ et $q^{\prime \prime}$ la fonction $q \longrightarrow \delta\left(q^{\prime}, q^{\prime \prime}, q\right)$ est bijective.

${ }^{2}$ Dans toutes les figures, le temps va vers le haut.
} 

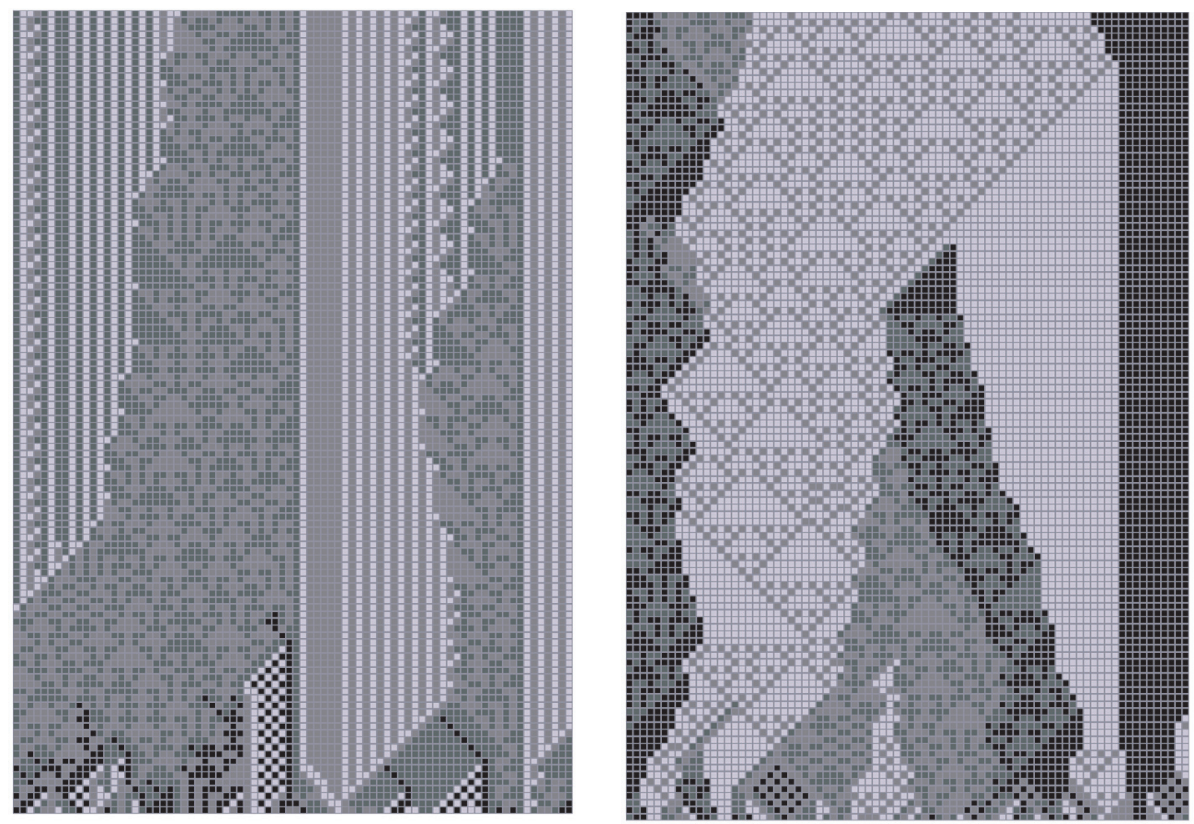

Figure 1. Exemple d'évolution d'automates captifs à quatre états à partir d'une configuration aléatoire pour la mesure de Bernouilli uniforme.

Partant de deux automates $\mathcal{A}$ et $\mathcal{B}$ dont les restrictions de $\delta_{\mathcal{A}}$ et $\delta_{\mathcal{B}}$ sont égales sur $Q_{\mathcal{A}} \cap Q_{\mathcal{B}}$, on peut obtenir un automate $\mathcal{A} \cup \mathcal{B}$ sur $Q_{\mathcal{A}} \cup Q_{\mathcal{B}}$ en spécifiant sa fonction locale de transition là où elle ne l'est pas déjà naturellement. Cela représente cependant une sorte de somme trop générale pour exhiber des propriétés vraiment intéressantes. L'idée est alors de diriger le choix de ces valeurs via un troisième automate $\mathcal{C}$ et d'obtenir ainsi une sorte de somme amalgamée. Plus précisément,

Définition 2. ${ }^{3}$ Soient deux automates $\mathcal{A}$ et $\mathcal{B}$ tels que $\delta_{\mathcal{A}}$ et $\delta_{\mathcal{B}}$ soient égales sur $Q_{\mathcal{A}} \cap Q_{\mathcal{B}}, \sigma_{\mathcal{A} \rightarrow \mathcal{B}}$ de $Q_{\mathcal{A}}$ dans $Q_{\mathcal{B}}$ et $\sigma_{\mathcal{B} \rightarrow \mathcal{A}}$ de $Q_{\mathcal{B}}$ dans $Q_{\mathcal{A}}$ des applications dont les restrictions à $Q_{\mathcal{A}} \cap Q_{\mathcal{B}}$ sont l'identité et soit $\mathcal{C}$ un troisième automate tel que $Q_{\mathcal{C}}=\{0,1\}$. On appelle foulard de $\mathcal{A}$ et $\mathcal{B}$ via $\mathcal{C}, \sigma_{\mathcal{A} \rightarrow \mathcal{B}}$ et $\sigma_{\mathcal{B} \rightarrow \mathcal{A}}$, l'automate noté $\mathcal{A} \oplus_{\mathcal{C}, \sigma_{\mathcal{A} \rightarrow \mathcal{B}}, \sigma_{\mathcal{B}} \rightarrow \mathcal{A}} \mathcal{B}$ :

- d'ensemble d'états $Q_{\mathcal{A}} \cup Q_{\mathcal{B}}$;

- de fonction locale $\delta$ définie ainsi : A tout triplet $\left(q_{1}, q_{2}, q_{3}\right)$ d'éléments de $Q_{\mathcal{A}} \cup Q_{\mathcal{B}}$, on fait correspondre le triplet $\left(\epsilon_{1}, \epsilon_{2}, \epsilon_{3}\right)$ de $Q_{\mathcal{C}}^{3}$ (qui est $\{0,1\}^{3}$ ) par $\epsilon_{i}=0 \Longleftrightarrow q_{i} \in Q_{\mathcal{A}}$ et $\epsilon_{i}=1 \Longleftrightarrow q_{i} \in Q_{\mathcal{B}}$.

La valeur 0 ou 1 de $\delta_{\mathcal{C}}\left(\epsilon_{1}, \epsilon_{2}, \epsilon_{3}\right)$ indique si $\delta\left(q_{1}, q_{2}, q_{3}\right)$ appartient à $Q_{\mathcal{A}}$ ou

\footnotetext{
${ }^{3}$ Remarquons que cette définition se généralise à la composition de $k$ automates via un automate à $k$ états, et que les définitions 1 et 2 peuvent se généraliser à tout voisinage.
} 

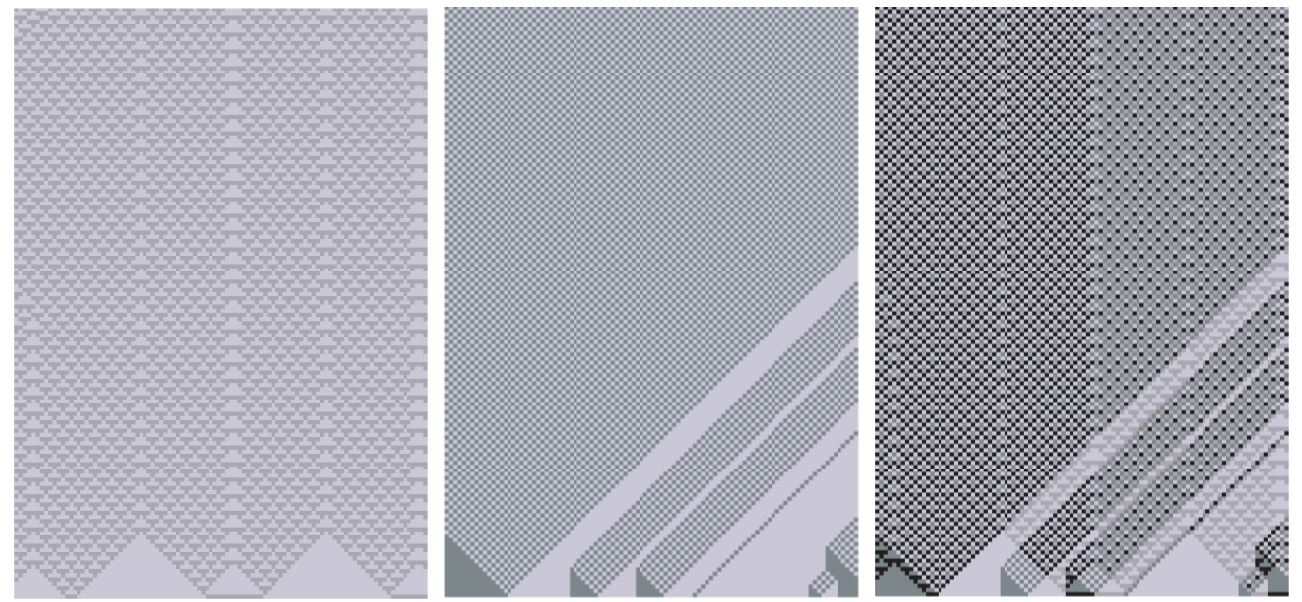

FiguRE 2. Évolution de l'automate $54 \oplus_{184, i d \text {, id }} 54$ (figure de droite) à partir d'une configuration aléatoire pour une mesure de Markov privilégiant l'appartenance au même sous-automate. Les deux copies de 54 sont disjointes et cet automate a quatre états. La figure centrale montre qu'en identifiant les états 0 et 1 d'une part et 2 et 3 d'autre part, on obtient une orbite de la règle 184 . La figure de gauche montre qu'en identifiant les états 0 et 2 d'une part et 1 et 3 d'autre part, on obtient une orbite de la règle 54 .

à $Q_{\mathcal{B}}$. Pour $q \in Q_{\mathcal{A}} \cup Q_{\mathcal{B}}$ on notera par $s_{\mathcal{A}}(q)$ (resp. $s_{\mathcal{B}}(q)$ ) l'état de $Q_{\mathcal{A}}$ (resp. $\left.Q_{\mathcal{B}}\right)$ qui est $q$ si $q \in Q_{\mathcal{A}}\left(\right.$ resp. $\left.q \in Q_{\mathcal{B}}\right)$ et $\sigma_{\mathcal{B} \rightarrow \mathcal{A}}(q)\left(\right.$ resp. $\left.\sigma_{\mathcal{A} \rightarrow \mathcal{B}}(q)\right)$ sinon.

Si $\delta_{\mathcal{C}}\left(\epsilon_{1}, \epsilon_{2}, \epsilon_{3}\right)=0, \delta\left(q_{1}, q_{2}, q_{3}\right)=\delta_{\mathcal{A}}\left(s_{\mathcal{A}}\left(q_{1}\right), s_{\mathcal{A}}\left(q_{2}\right), s_{\mathcal{A}}\left(q_{3}\right)\right)$.

Si $\delta_{\mathcal{C}}\left(\epsilon_{1}, \epsilon_{2}, \epsilon_{3}\right)=1, \delta\left(q_{1}, q_{2}, q_{3}\right)=\delta_{\mathcal{B}}\left(s_{\mathcal{B}}\left(q_{1}\right), s_{\mathcal{B}}\left(q_{2}\right), s_{\mathcal{B}}\left(q_{3}\right)\right)$.

Bien sûr, si on choisit $\mathcal{C}$ bijectif et des permutations adéquates, on retrouve la notion de «partiellement commutatif » de [8]. Observons que, connaissant $\mathcal{A}$ et $\mathcal{B}$,

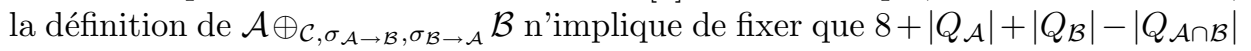
valeurs. Dans la suite on supposera, par souci de simplicité (cette condition étant toujours réalisée si on considère $\mathcal{C}^{2}$ ), que $\delta_{\mathcal{C}}(0,0,0)=0$ et $\delta_{\mathcal{C}}(1,1,1)=1$ (i.e. $\mathcal{C}$ est captif).

La Figure 2 montre une évolution de $54 \oplus_{184, i d, \text { id }} 54$ ainsi que deux orbites que l'on peut obtenir à partir de cette dernière par projections. Elle donne une idée de ce que peut apporter ce type de construction. Plus généralement les Figures 1 et 2 suggèrent que les deux classes introduites peuvent fournir des exemples d'automates avec auto-organisation, qui apparaissent rarement par génération aléatoire, et par conséquent rendre éventuellement compte d'un tel phénomène. Notons que 

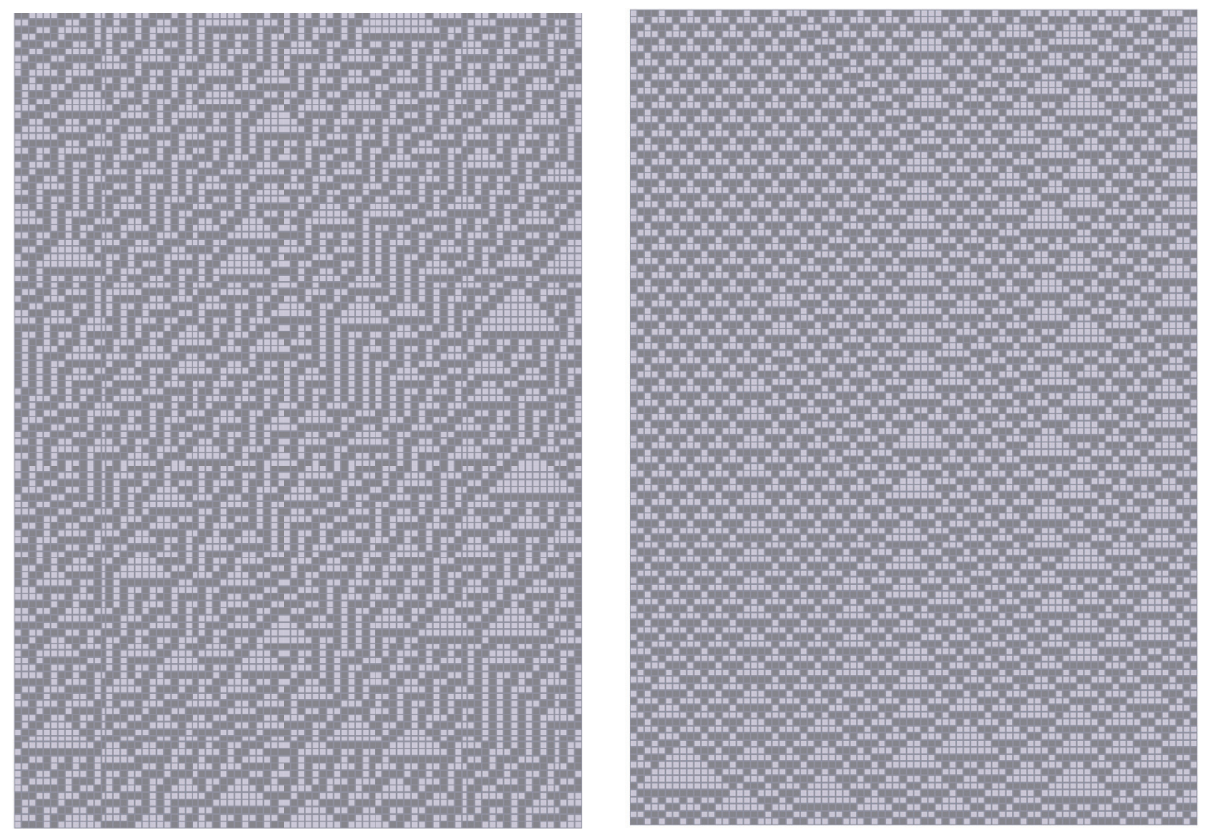

Figure 3. Exemple d'évolution des règles 30 (sans autoorganisation) et 54 (avec auto-organisation) à partir d'une configuration aléatoire pour la mesure de Bernouilli uniforme.

nous n'avons pas de définition de l'auto-organisation mais dire qu'un automate cellulaire a la propriété d'auto-organisation signifie, ici pour nous, que presque tous ses diagrammes espace-temps sont constitués de larges plages stables (fonds) séparées par des frontières (particules). Cette notion d'auto-organisation n'a jamais été définie formellement; elle résulte de l'observation de création de particules $[1,33]$ qui peuvent avoir un comportement simple rationnel [26] ou même engendrer des marches aléatoires [9-12]. Depuis les études empiriques de [13,14], elle a conduit à la théorie de la «mécanique du calcul » de Crutchfield, Hansen et leurs collaborateurs [4-7, 15, 19,20,42]. Enfin, plus récemment une étude formelle a été entreprise par Pivato [39-41].

La suite de cet article se compose donc d'un premier paragraphe consacré à quelques rappels de notions et résultats nécessaires pour étudier et discuter de façon plus détaillée les automates cellulaires captifs et les foulards. Nous évoquerons en particulier les notions de sous-automate et d'automate-quotient, des relations de préordres que nous leurs associons par des méthodes de groupage et qui conduisent à des classifications algébriques des automates cellulaires. Puis deux paragraphes seront dédiés respectivement à la classe des automates cellulaires captifs et à celle des foulards. 


\section{Classifications algÉBriques}

La classification empirique de Wolfram [47] exhibe quatre classes. Les deux premières contiennent les automates partout ou presque partout nilpotents, dont la dynamique est assez simple. Les deux dernières contiennent les automates dont la dynamique est dite chaotique et qui se distinguent essentiellement par le fait qu'apparaît (classe 4) ou non (classe 3) de l'auto-organisation dans leurs évolutions, voir la Figure 3. Les classifications ultérieures n'ont pas rendu compte de ce phénomène d'auto-organisation. Pas même la classification de Kůrka [21] que nous rappelons maintenant.

Dans ce cadre un automate cellulaire $\mathcal{A}$ est vu comme le système dynamique discret $\left(\Delta_{\mathcal{A}}, Q_{\mathcal{A}}^{\mathbb{Z}}\right), Q_{\mathcal{A}}^{\mathbb{Z}}$ est muni de la topologie de Tychonoff et d'une distance définie par : $c$ et $c^{\prime} \in Q_{\mathcal{A}}^{\mathbb{Z}}$ et $d\left(c, c^{\prime}\right)=\sum_{z \in \mathbb{Z}} \frac{\sharp\left(c(z), c^{\prime}(z)\right)}{2^{|z|+1}}$ où, pour $q, q^{\prime} \operatorname{de} Q_{\mathcal{A}}, \sharp\left(q, q^{\prime}\right)=1$ si $q \neq q^{\prime}$ et 0 sinon. Quatre classes d'automates [21] peuvent alors être distinguées : les automates équicontinus partout (l'identité), ceux qui ont seulement des points d'équicontinuité (règle 109), les automates sensibles aux conditions initiales non expansifs (règle 60), les expansifs (règle 90) . Ces caractéristiques topologiques $^{5}$ ne rendent pas complètement compte de l'auto-organisation dans la mesure où les automates qui présentent cette distinction figurent avec d'autres dans la classe des sensibles dans la classification de Kůrka [?]. Cependant, une approche combinant topologie et mesures rend compte d'une certaine forme macroscopique d'autoorganisation $[17,18,22-24]$.

Afin, entre autres, de tenter de mieux cerner ce phénomène, un autre point de vue a été introduit $[27,29,34,43,46]$.

\subsection{Sous-automate et Automate projeté D'Un AUtomate Cellulaire}

L'idée de considérer un sous-automate d'un automate est assez naturelle d'autant que les fonds dûs à l'auto-organisation sont souvent des sous-automates. Mais la Figure 2 suggère d'observer ce qui se passe lorsque l'on identifie certains états d'un automate. Apparaît alors une notion d'automate projection.

\section{Définition 3.}

(1) Un automate $\mathcal{A}=\left(Q_{\mathcal{A}}, \delta_{\mathcal{A}}\right)$ est un sous-automate d'un automate $\mathcal{B}=$ $\left(Q_{\mathcal{B}}, \delta_{\mathcal{B}}\right)$, noté $\mathcal{A} \sqsubseteq \mathcal{B}$, s'il existe une injection $\varphi$ de $Q_{\mathcal{A}}$ dans $Q_{\mathcal{B}}$ telle que pour tous $q_{1}, q_{2}, \bar{q}_{3}, \varphi\left(\delta_{\mathcal{A}}\left(q_{1}, q_{2}, q_{3}\right)\right)=\delta_{\mathcal{B}}\left(\varphi\left(q_{1}\right), \varphi\left(q_{2}\right), \varphi\left(q_{3}\right)\right)$ (i.e. $\mathcal{A}$ est un sous-système de $\mathcal{B})$.

\footnotetext{
${ }^{4} \mathcal{A}$ est équicontinu en $c$ quand l'orbite de toute configuration $c^{\prime}$ près de $c$ reste près de $c$ i.e. : $\forall \epsilon>0, \exists \delta_{c, \epsilon}, \forall c^{\prime} \in Q_{\mathcal{A}}^{\mathbb{Z}}, d\left(c, c^{\prime}\right)<\delta_{c, \epsilon}$ implique, $\forall t \in \mathbb{N}, d\left(\Delta_{\mathcal{A}}^{t}(c), \Delta_{\mathcal{A}}^{t}\left(c^{\prime}\right)\right)<\epsilon$.

$\mathcal{A}$ est sensible quand, uniformément, pour toute configuration $c$ il existe des configurations $c^{\prime}$ arbitrairement proches de $c$ qui s'éloignent de $c$ lors de l'évolution. i.e. : $\exists \epsilon>0, \forall c \in Q_{\mathcal{A}}, \forall \delta>0$, $\exists c^{\prime} \in Q_{\mathcal{A}}, d\left(c, c^{\prime}\right)<\delta$ et $\exists t \in \mathbb{N}, d\left(\Delta_{\mathcal{A}}^{t}(c), \Delta_{\mathcal{A}}^{t}\left(c^{\prime}\right)\right) \geq \epsilon$.

$\mathcal{A}$ est expansif quand toutes les différences entre deux configurations sont amplifiées par l'évolution i.e. : $\exists \epsilon>0, \forall c \in Q_{\mathcal{A}}, \forall c^{\prime} \in Q_{\mathcal{A}}, c \neq c^{\prime}, \exists t \in \mathbb{N}, d\left(\Delta_{\mathcal{A}}^{t}(c), \Delta_{\mathcal{A}}^{t}\left(c^{\prime}\right)\right) \geq \epsilon$.

${ }^{5}$ Notons qu'une étude (de ce point de vue) complète des automates à deux états se trouve dans [3].
} 
(2) Un automate $\mathcal{A}$ est le projeté d'un automate $\mathcal{B}$ par $\varsigma$, noté $\mathcal{A} \unlhd_{\varsigma} \mathcal{B}$ ou $\mathcal{A} \unlhd \mathcal{B}$, si

- $\varsigma$ est une surjection de $Q_{\mathcal{B}}$ sur $Q_{\mathcal{A}}$;

- et, $\forall q_{1}^{\mathcal{A}}, q_{2}^{\mathcal{A}}, q_{3}^{\mathcal{A}} \in Q_{\mathcal{A}}, \forall q_{1}^{\mathcal{B}}, q_{2}^{\mathcal{B}}, q_{3}^{\mathcal{B}} \in Q_{\mathcal{B}}, \varsigma\left(q_{1}^{\mathcal{B}}\right)=q_{1}^{\mathcal{A}}$ et $\varsigma\left(q_{2}^{\mathcal{B}}\right)=q_{2}^{\mathcal{A}}$ et $\varsigma\left(q_{3}^{\mathcal{B}}\right)=q_{3}^{\mathcal{A}}$ impliquent $\varsigma\left(\delta_{\mathcal{B}}\left(q_{1}^{\mathcal{B}}, q_{2}^{\mathcal{B}}, q_{3}^{\mathcal{B}}\right)\right)=\delta_{\mathcal{A}}\left(q_{1}^{\mathcal{A}}, q_{2}^{\mathcal{A}}, q_{3}^{\mathcal{A}}\right)$.

(i.e. $\mathcal{A}$ est un facteur de $\mathcal{B})$.

Ainsi (voir Fig. 2), dans le cas de l'automate $54 \oplus_{184, i d, i d} 54$, l'automate 54 est à la fois sous-automate et projeté de $54 \oplus_{184, i d, \text { id }} 54$ tandis que 184 est projeté mais pas sous-automate de $54 \oplus_{184, i d, \text { id }} 54$.

\subsection{Automates Cellulaires Groupés}

Lorsqu'on observe naïvement une orbite, on observe de petits carrés de couleur. Sans changer l'objet d'étude ('ensemble des orbites d'un automate cellulaire), on peut changer le mode d'observation et considérer que le diagramme espace-temps est pavé par de grands carrés, de grands rectangles ou encore de grands rectangles décalés ${ }^{6}$. Ces remarques ont été formalisées dans [29, 34, 43]. On obtient alors une nouvelle notion, celle d'automate groupé.

\section{Définition 4.}

(1) À tout automate $\mathcal{A}=\left(Q_{\mathcal{A}}, \delta_{\mathcal{A}}\right)$ et à tout entier $n \in \mathbb{N}^{\star}$, on fait correspondre l'automate $o_{n}(\mathcal{A})=\left(Q_{o_{n}(\mathcal{A})}=Q_{\mathcal{A}}^{n}, \delta_{o_{n}(\mathcal{A})}\right)$ avec

$\delta_{o_{n}(\mathcal{A})}\left(\left(q_{1}^{g}, \ldots, q_{n}^{g}\right),\left(q_{1}^{c}, \ldots, q_{n}^{c}\right),\left(q_{1}^{d}, \ldots, q_{n}^{d}\right)\right)=\left(\delta_{\mathcal{A}}\left(q_{n}^{g}, q_{1}^{c}, q_{2}^{c}\right), \ldots, \delta_{\mathcal{A}}\left(q_{n-1}^{c}, q_{n}^{c}, q_{1}^{d},\right)\right)$.

(2) À tout automate $\mathcal{A}=\left(Q_{\mathcal{A}}, \delta_{\mathcal{A}}\right)$ et à tous entiers $n, m \in \mathbb{N}^{\star}$ et $s \in \mathbb{Z}$, on fait correspondre l'automate $\mathcal{A}^{(n, p, s)}$, groupé de $\mathcal{A}$ comme étant l'automate de voisinage $\{-1,0,+1\}$ de fonction globale $\sigma^{s} \circ o_{n}^{-1} \circ \Delta_{o_{n}(\mathcal{A})}^{p} \circ o_{n}(\sigma$ est le décalage à droite et $o_{n}^{-1}$ est l'inverse de l'extension naturelle de $o_{n}$ comme fonction de $Q_{\mathcal{A}}^{\mathbb{Z}}$ dans $\left.\left(Q_{\mathcal{A}}^{n}\right)^{\mathbb{Z}}\right)$.

Par le théorème de Hedlund [16], $\mathcal{A}^{(n, p, s)}$ est un automate cellulaire à $Q_{\mathcal{A}}^{n}$ états de voisinage $V \subseteq\{-v, \ldots,+w\}$, qui peut être vu comme un automate à $Q_{\mathcal{A}}^{n h}$ états de voisinage $\{-1,0,+1\}$ si $v+w+1 \leq 3 h$.

\subsection{ORDRES SUR LES ClASSES D'AUtOMATES}

Les notions introduites précédemment permettent de définir sur la classe des automates cellulaires des relations $\square_{\square}, \emptyset_{\diamond}, \unlhd_{\square}, \unlhd_{\diamond}, \unlhd \sqsubseteq \square$ et $\unlhd \sqsubseteq \diamond$ comme suit. Si $\mathcal{A}$ et $\mathcal{B}$ sont deux automates :

\section{Définition 5.}

(1) $\mathcal{A} \sqsubseteq \square \mathcal{B}$ s'il existe deux entiers $n_{\mathcal{A}}$ et $n_{\mathcal{B}}$ de $\mathbb{N}^{\star}$ tels que $\mathcal{A}^{\left(n_{\mathcal{A}}, n_{\mathcal{A}}, 0\right)}$ est un sous-automate de $\mathcal{B}^{\left(n_{\mathcal{B}}, n_{\mathcal{B}}, 0\right)}$, soit $\mathcal{A}^{\left(n_{\mathcal{A}}, n_{\mathcal{A}}, 0\right)} \sqsubseteq \mathcal{B}^{\left(n_{\mathcal{B}}, n_{\mathcal{B}}, 0\right)}$.

\footnotetext{
${ }^{6}$ D'autres exemples de pavages de l'espace-temps se trouvent en [30-32,37].
} 

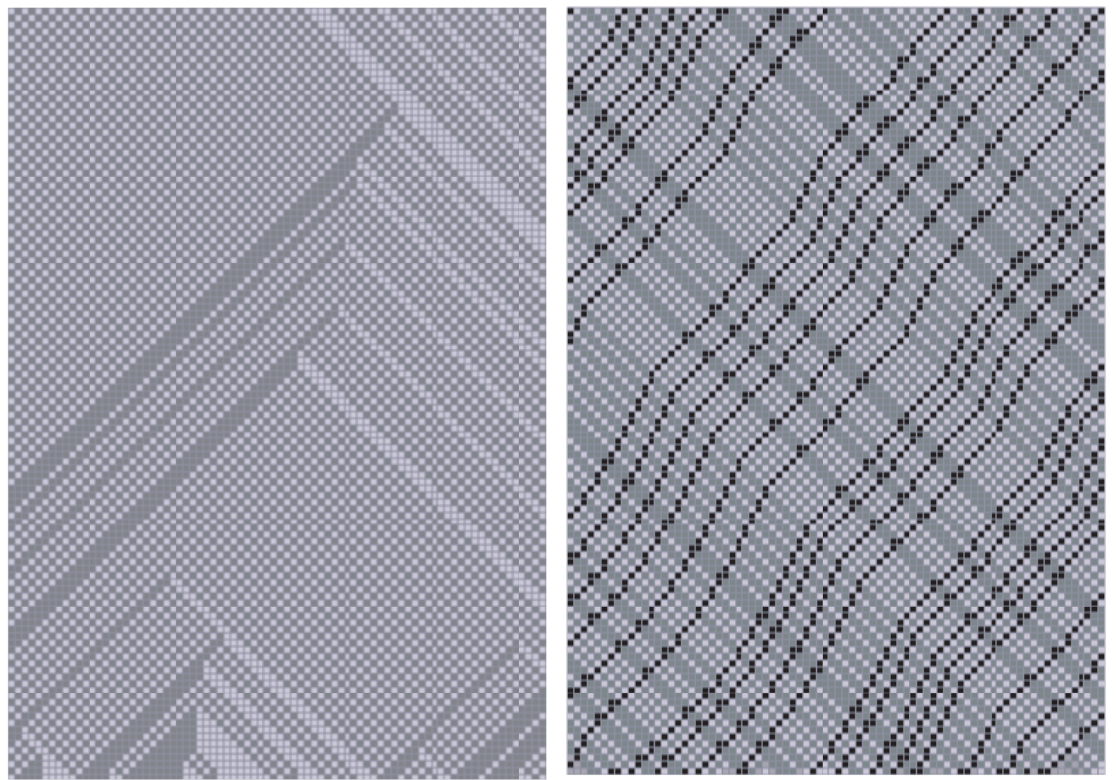

Figure 4. Une orbite de l'automate 184 (à gauche) et de $160 \oplus_{186,0-0,0-0} 84$ (à droite) pour la mesure de Bernouilli uniforme.

(2) $\mathcal{A} \sqsubseteq \diamond \mathcal{B}$ s'il existe six entiers $n_{\mathcal{A}}, n_{\mathcal{B}}, p_{\mathcal{A}}, p_{\mathcal{B}}, s_{\mathcal{A}}, s_{\mathcal{B}}$ de $\mathbb{N}^{\star}$ ou de $\mathbb{Z}$ tels que $\mathcal{A}^{\left(n_{\mathcal{A}}, p_{\mathcal{A}}, s_{\mathcal{A}}\right)} \sqsubseteq \mathcal{B}^{\left(n_{\mathcal{B}}, p_{\mathcal{B}}, s_{\mathcal{B}}\right)}$.

(3) $\mathcal{A} \unlhd_{\square} \mathcal{B}$ s'il existe deux entiers $n_{\mathcal{A}}$ et $n_{\mathcal{B}}$ de $\mathbb{N}^{\star}$ tels que $\mathcal{A}^{\left(n_{\mathcal{A}}, n_{\mathcal{A}}, 0\right)}$ soit un projeté de $\mathcal{B}^{\left(n_{\mathcal{B}}, n_{\mathcal{B}}, 0\right)}$, soit $\mathcal{A}^{\left(n_{\mathcal{A}}, n_{\mathcal{A}}, 0\right)} \unlhd \mathcal{B}^{\left(n_{\mathcal{B}}, n_{\mathcal{B}}, 0\right)}$.

(4) $\mathcal{A} \unlhd_{\diamond} \mathcal{B}$ s'il existe six entiers $n_{\mathcal{A}}, n_{\mathcal{B}}, p_{\mathcal{A}}, p_{\mathcal{B}}, s_{\mathcal{A}}, s_{\mathcal{B}}$ de $\mathbb{N}^{\star}$ ou de $\mathbb{Z}$ tels que $\mathcal{A}^{\left(n_{\mathcal{A}}, p_{\mathcal{A}}, s_{\mathcal{A}}\right)} \unlhd \mathcal{B}^{\left(n_{\mathcal{B}}, p_{\mathcal{B}}, s_{\mathcal{B}}\right)}$.

$\mathcal{A} \sqsubseteq \square \mathcal{B}($ ou $\mathcal{A} \sqsubseteq \diamond \mathcal{B})$ peut s'interpréter comme le fait que, à groupage près c'està-dire à un changement d'échelle près, toutes les orbites de $\mathcal{A}$ figurent parmi les orbites de $\mathcal{B}$. De façon analogue, $\mathcal{A} \unlhd_{\square} \mathcal{B}$ (ou $\mathcal{A} \unlhd_{\diamond} \mathcal{B}$ ) peut s'interpréter comme le fait que, à groupage près, toutes les orbites de $\mathcal{A}$ peuvent être obtenues à partir des orbites de $\mathcal{B}$ par identification d'états. Ainsi l'évolution de 184 (Fig. 4) montre que les décalages à gauche et à droite sont des sous-automates du $(3,3,0)$-groupé de 184 , mais pas de 184 non groupée. De même la règle qui envoie $(x, 0, y)$ sur 0 et $(x, 1, y)$ sur 1 (identité) est un sous-automate du $(3,3,3)$-groupé de 184 , alors qu'elle n'est un sous-automate ni de 184 non groupée, ni du (3, 3, 0)-groupé de 184 . Par ailleurs, sur la même Figure 4, peut-on observer que 186 et le décalage à gauche sont des projetés de $160 \oplus_{186,0-0,0-0} 84^{7}$ sans groupage.

\footnotetext{
${ }^{7}$ Les applications $\sigma_{\mathcal{A} \rightarrow \mathcal{B}}$ et $\sigma_{\mathcal{B} \rightarrow \mathcal{A}}$ sont notées $a_{0}-a_{1}-\ldots$ avec $a_{0}=\sigma_{\mathcal{A} \rightarrow \mathcal{B}}(0), a_{1}=\sigma_{\mathcal{A} \rightarrow \mathcal{B}}(1)$, $\ldots$ ou $a_{0}=\sigma_{\mathcal{B} \rightarrow \mathcal{A}}(0), a_{1}=\sigma_{\mathcal{B} \rightarrow \mathcal{A}}(1), \ldots$
} 
On peut également les combiner et définir $\unlhd \sqsubseteq$ « être le projeté d'un sousautomate $»$ dans les deux cas $\square$ et $\diamond$ de la Définition 5 . Chacune de ces relations est une relation de préordre ${ }^{8}$ et définit rigoureusement une notion de simulation, ce qui, nous y reviendrons plus loin, permet de donner un sens précis à une notion d'universalité intrinsèque associée.

Chacun de ces préordres induit un ordre sur les classes de l'équivalence qui lui est canoniquement associée. Les ordres obtenus, non totaux, représentent des classifications (que nous appelons algébriques) avec une infinité de classes. Chaque ordre indexé par $\diamond$ raffine l'ordre indexé par $\square$ correspondant. Il est prouvé dans [46] que tout ordre $\unlhd \sqsubseteq$ est plus fin que les ordres $\sqsubseteq$ et $\unlhd$ correspondants qui sont incomparables entre eux. Ils ont tous un plus petit élément (la classe de l'automate à un état). Ont-ils un plus grand élément? La réponse dépend de l'ordre (Fig. 5). Elle est négative pour l'ordre sous-automate dans le groupage carré (ㄷ) [29,43] et positive pour les ordres sous-automate dans le groupage rectangle [35,36] (et par conséquent pour le groupage $\unlhd \sqsubseteq$ ). Elle est ouverte pour l'ordre $\unlhd$. Ce qui est ici remarquable, c'est que la classe maximum pour l'ordre sous-automate dans le groupage rectangle, est exactement la classe des automates que l'on nomme intrinsèquement universels, c'est-à-dire des automates qui simulent tous les autres au sens du préordre correspondant. Il est à noter que l'intrinsèque universalité est une notion plus forte que celle d'universalité pour le calcul (Turing-universalité) puisqu'il existe des automates calculateurs universels (en utilisant la construction de [44]) qui ne sont pas intrinséquement universels [34,46].

Notons qu'il est prouvé dans [46] que presqu'aucun automate cellulaire n'a de sous-automate non trivial. Cela signifie en particulier que les classes correspondant à ces automates dans les groupages $\square$ et $\diamond$ se situent juste au-dessus de leur minimum. Il est de plus prouvé que, si l'on exclue ce minimum, elles sont minimales pour ces ordres, c'est-à-dire qu'elles sont au premier niveau de chacune de ces hiérarchies. Il s'en suit que les classes qui sont au-dessus de ce premier niveau sont les classes d'automates ayant des sous-automates non triviaux, donc d'automates capables d'auto-organisation (qui, selon le point de vue ici adopté, correspond à la présence de «fonds », issus de sous-automates, dans lesquels évoluent des particules).

Voyons maintenant si, et alors comment, les automates captifs et les automates foulards permettent de progresser dans la connaissance de ces automates complexes que sont les automates cellulaires qui ont la propriété d'auto-organisation.

\section{Automates Captifs}

Les automates captifs on été étudiés dans [46]. Ils répondent à quelques-unes de nos attentes.

Si l'on se place du point de vue des classes d'automates caractérisées par une propriété de leur table de transition locale, ils se distinguent notablement des deux

\footnotetext{
${ }^{8} \mathrm{La}$ relation $\sqsubseteq \unlhd$, à savoir être sous-automate d'un projeté, n'est pas un préordre, donc ne nous intéresse pas ici. En outre, la relation $\sqsubseteq \unlhd \sqsubseteq$ n'est autre que $\unlhd \sqsubseteq$.
} 

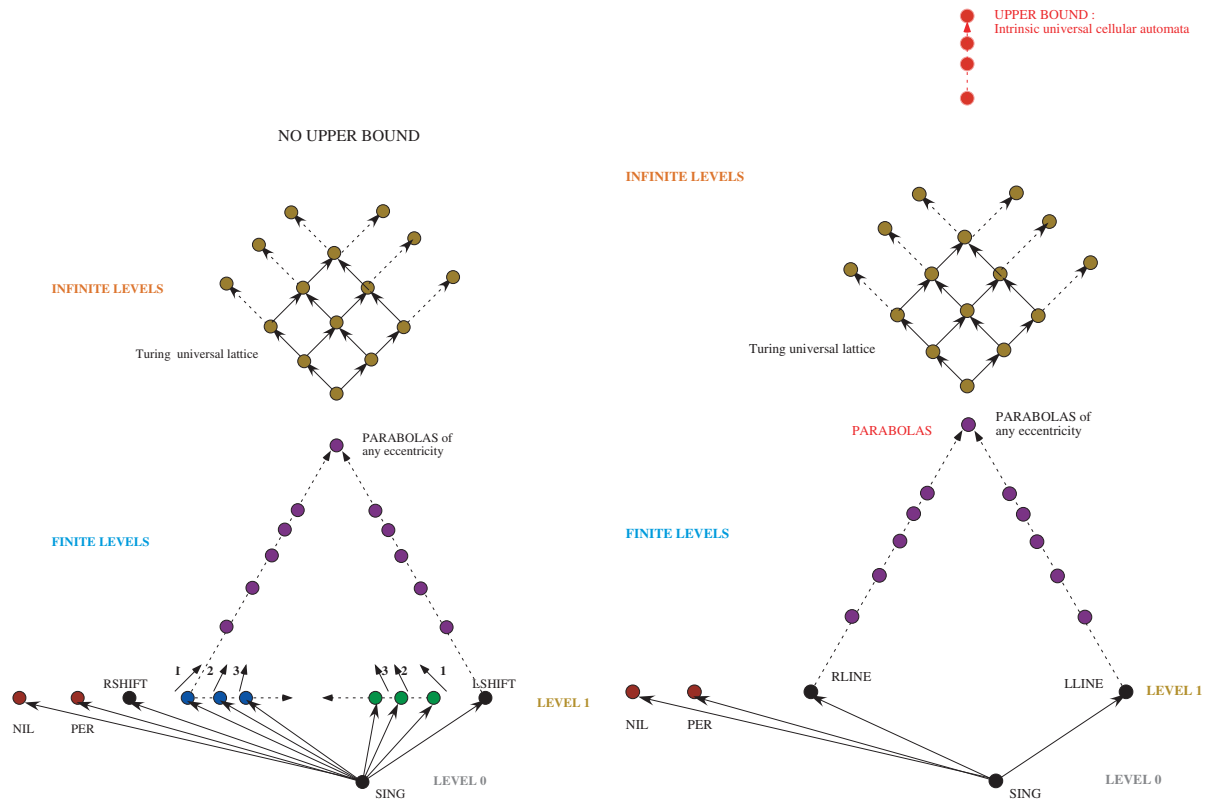

FiguRE 5. Les ordres $\sqsubseteq \square$ et $\sqsubseteq \diamond$.

classes évoquées dans l'introduction. En effet, les automates cellulaires additifs captifs ayant strictement plus de deux états sont les translations ou une fonction constante égale à l'élément neutre du groupe. De plus, si un automate captif est permutif en deux positions distinctes, alors il possède seulement deux états.

Il est également prouvé que, pour tout automate cellulaire $\mathcal{A}$, il existe un automate captif $\mathcal{C}$ tel que $\mathcal{A} \sqsubseteq \diamond \stackrel{\mathcal{C}}{\mathcal{A}}$ (où $\mathcal{A}$ dénote la classe d'équivalence de $\mathcal{A}$ ). Cette dernière propriété implique l'existence d'automates cellulaires intrinsèquement universels (pour la simulation en question) captifs [45]. De plus, il est prouvé que presque tous les automates cellulaires captifs sont intrinsèquement universels à partir d'une notion de densité définie comme suit : soit $\mathcal{E}$ un ensemble d'automates cellulaires et $\mathcal{P}$ une propriété d'automates cellulaires, alors, la densité dans $\mathcal{E}$ des automates ayant la propriété $\mathcal{P}$ est la limite, lorsqu'elle existe, $\lim _{n \rightarrow \infty} \frac{\left|\mathcal{E}_{n} \cap \mathcal{P}\right|}{\left|\mathcal{E}_{n}\right|}$ où $\mathcal{E}_{n}$ est l'ensemble des éléments de $\mathcal{E}$ à $n$ états. Cependant il est indécidable de savoir si un automate cellulaire captif est intrinsèquement universel.

D'un point de vue dynamique, il n'existe qu'une seule classe (pour $\sim \diamond$ ) d'un automate captif expansif : celle du décalage. Et il n'est pas difficile de construire des automates captifs dans chaque autre classe de la classification de Kơrka.

On peut donc en conclure que, d'une certaine manière, cette classe est assez représentative des automates cellulaires en général et qu'une étude plus systématique, exploitant de nombreuses observations empiriques et le fait que de tels automates sont plutôt faciles à construire, peut ouvrir des perspectives pertinentes sur le monde complexe des automates cellulaires. En particulier sur l'auto-organisation. 

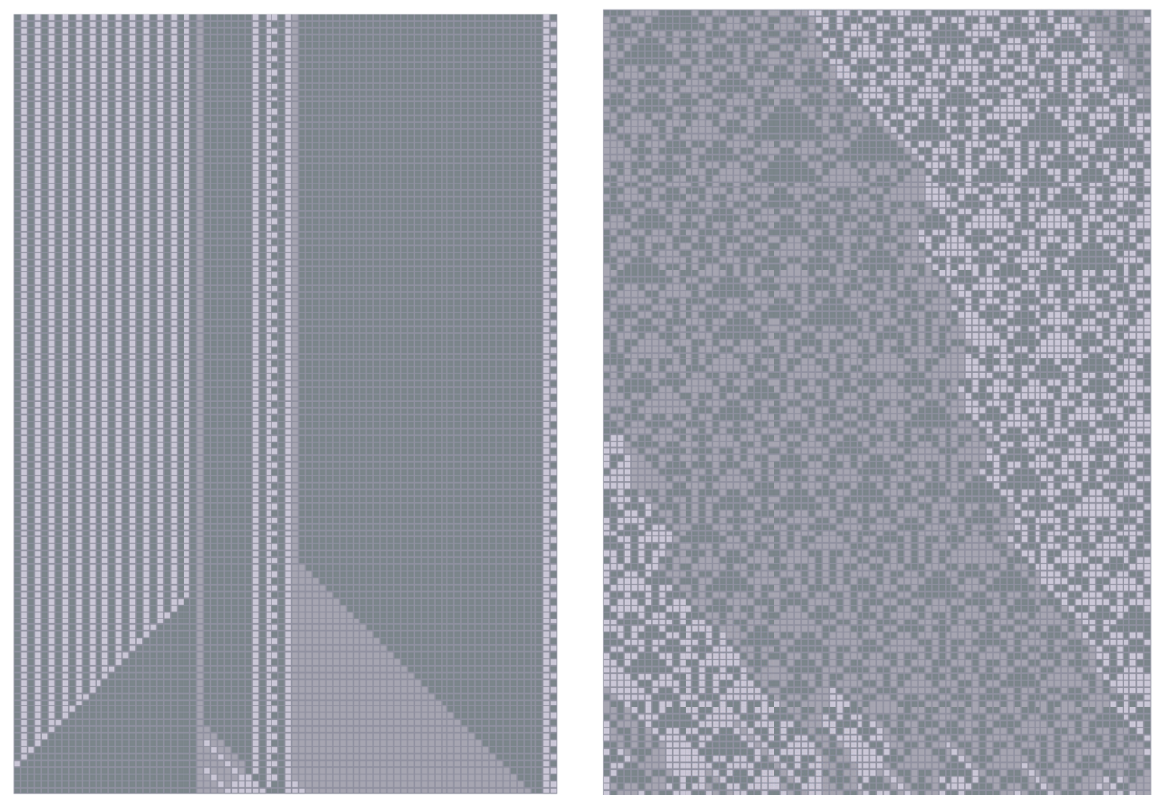

FiguRE 6. Exemple d'évolution d'automates captifs.

Il est possible en effet d'obtenir des marches aléatoires, entre autres des diagrammes espace-temps composés de plages qui semblent représenter des luttes d'influence de sous-automates $[9,12]$, voir la Figure 6.

\section{Automates foulards}

Les automates foulards sont encore peu étudiés. Ils nous semblent cependant riches de potentialités à explorer. Tentons de le montrer sur un exemple.

\subsection{UN EXEMPLE}

L'observation de l'évolution de la règle 242 de la Figure 7 conduit à penser que deux automates sont à l'œuvre, dont les plages d'expression sont séparées par des diagonales vers la droite. Cet automate ne peut être un foulard qui devrait, dans ce cas avoir au moins trois états, mais pourrait-il se rapporter de quelque manière à un foulard amalgamant ces deux automates apparents? De fait, on a le résultat suivant.

Proposition. Il existe une projection $\varsigma$ telle que toute orbite de la règle $242^{(3,3,0)}$ est l'image par $\varsigma$ d'une orbite de $114 \oplus_{240,1-1,1-1} 254^{(3,3,0)}$. 

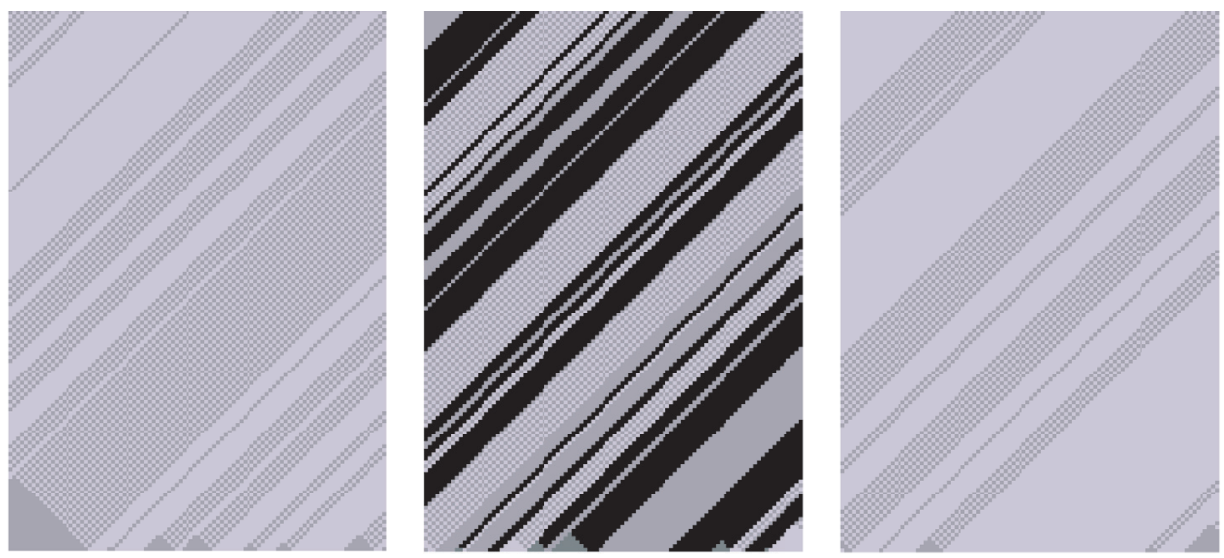

Figure 7. Exemple d'évolution des automates 242, $114 \oplus_{240,1-1,1-1} 254$ et $\varsigma\left(114 \oplus_{240,1-1,1-1} 254\right)$ (avec $\varsigma$ égal à 0 sauf $\varsigma(0)=1$ ) sur une ligne aléatoire de mesure markovienne.

Lemme 1. Soient $\mathcal{A}$ et $\mathcal{B}$ des automates quelconques. Le $(3,3,0)$-groupé de $\mathcal{A} \oplus_{240}$, ${ }_{1-1,1-1} \mathcal{B}$ admet comme sous-automate l'automate $\mathcal{S}=\left(Q_{\mathcal{S}}, \delta_{\mathcal{S}}\right)$ où $Q_{\mathcal{S}}=\left\{\left(q_{1}, q_{2}\right.\right.$, $\left.q_{3}\right),\left(q_{1}, q_{2}^{\prime}, q_{3}^{\prime}\right),\left(q_{1}^{\prime}, q_{2}^{\prime}, q_{3}\right),\left(q_{1}^{\prime}, q_{2}^{\prime}, q_{3}^{\prime}\right) / q_{i} \in Q_{\mathcal{A}}$ et $\left.q_{i}^{\prime} \in Q_{\mathcal{B}}\right\}$ et $\delta_{\mathcal{S}}$ l'injection canonique de $Q_{\mathcal{S}}$ dans l'ensemble des états de $\left(\mathcal{A} \oplus_{240,1-1,1-1} \mathcal{B}\right)^{(3,3,0)}$.

Il suffit d'observer que la règle 240 est le décalage à droite. Le sous-automate $\mathcal{S}$ va nous permettre d'éliminer les configurations de $114 \oplus_{240,1-1,1-1} 254$ qui possèdent un seul état de $\mathcal{B}$ entouré d'états de $\mathcal{A}$.

Remarque. Une configuration non triviale de $\mathcal{S}$, lorsqu'elle est dégroupée par $o_{3}^{-1}$ est constituée d'intervalles de longueur quelconque d'états de $Q_{\mathcal{A}}$ et d'intervalles d'états de $Q_{\mathcal{B}}$ de longueur nécessairement supérieure ou égale à deux. Une configuration non triviale de 242 se découpe en intervalles de deux types : ceux qui sont bordés de 0 et ne contiennent pas d'occurrence de 11 (typa a) et ceux qui ne sont constitués que de 1 et contiennent au moins une occurrence de 11 (type b).

Il en résulte le lemme suivant.

Lemme 2. Il existe une injection $\phi$ des configurations de 242 dans les configurations ouvertes par $o_{3}^{-1}$ de $\mathcal{S}$.

Supposons que $Q_{\mathcal{A}}=\{0,1\}$ et $Q_{\mathcal{B}}=\{2,3\}$. Alors l'application $\xi$ définie par $\xi(0)=1, \xi(1)=0$ et $\xi(2)=\xi(3)=0$ induit évidemment une application $\varsigma$ de $Q_{\mathcal{S}}$ sur $\{0,1\}^{3}$ par $\varsigma\left(q_{1}, q_{2}, q_{3}\right)=\left(\xi\left(q_{1}\right), \xi\left(q_{2}\right), \xi\left(q_{3}\right)\right)$ qui est surjective.

Rappelons que les transitions d'états de 242 sont :

$$
\begin{gathered}
\delta(0,0,0)=0, \delta(0,0,1)=1 \delta(0,1,0)=0, \delta(0,1,1)=0, \\
\delta(1,0,0)=1, \delta(1,0,1)=1, \delta(1,1,0)=1, \delta(1,1,1)=1,
\end{gathered}
$$

que la règle 114 est la règle 242 avec $\delta(1,1,1)=0$ et que la règle 254 a toujours pour image 1 sauf 000 qui donne 0 . 
Considérons trois états $\left(\theta_{1}^{\prime}, \theta_{2}^{\prime}, \theta_{3}^{\prime}\right),\left(\theta_{1}, \theta_{2}, \theta_{3}\right)$ et $\left(\theta_{1}^{\prime \prime}, \theta_{2}^{\prime \prime}, \theta_{3}^{\prime \prime}\right)$ de $\mathcal{S}$ qui sont des images par l'injection $\phi$ d'états de $242^{(3,3,0)}$ et discutons la présence d'états de 254 dans le motif $m=\theta_{3}^{\prime} \theta_{1} \theta_{2} \theta_{3} \theta_{1}^{\prime \prime}$ :

- Aucun état de 254 n'apparaît. Alors, en une étape, $\delta_{114 \oplus_{240,1-1,1-1} 254}$ utilise la règle 114 et comme le motif $\left(\theta_{1}^{\prime}, \theta_{2}^{\prime}, \theta_{3}^{\prime}, \theta_{1}, \theta_{2}, \theta_{3}, \theta_{1}^{\prime \prime}, \theta_{2}^{\prime \prime}, \theta_{3}^{\prime \prime}\right)$ est l'image par $\phi$ d'états de $242^{(3,3,0)}$, le motif $\xi(m)$ ne contient pas le sous-motif 11 . Donc 242 utilise sur $\xi(m)$ les mêmes transitions que 114.

- Un seul état de 254 apparaît. Par définition de $\mathcal{S}$ il apparaît soit en position $\theta_{3}^{\prime}$ et alors (encore par définition de $\mathcal{S}$ ) $\theta_{2}^{\prime}$ est un état de 254 , soit en position $\theta_{1}^{\prime \prime}$ et alors $\theta_{2}^{\prime \prime}$ est un état de 254. Comme les règles sont invariantes par décalage, on se ramène au cas où deux états de 254 apparaissent.

- Exactement deux états de 254 apparaissent. Par définition de $\mathcal{S}$, ils sont consécutifs.

Examinons d'abord le motif $\mu=\epsilon_{1} \epsilon_{2} 0 b b(\epsilon \in\{0,1\}$ et $b \in\{2,3\})$. Par 242, $\varsigma(\mu)$ donne $\left(\delta_{242}\left(\epsilon_{1}, \epsilon_{2}, 1\right) \delta_{242}\left(\epsilon_{2}, 1,0\right) 0\right)$ qui est $\left(\delta_{114}\left(\epsilon_{1}, \epsilon_{2}, 1\right)\right.$ $\left.\delta_{114}\left(\epsilon_{2}, 1,0\right) 1\right)$ puisque $\epsilon_{1}, \epsilon_{2} \neq 11$. Par $114 \oplus_{240,1-1,1-1} 254$, on obtient le même résultat.

On raisonne de façon identique pour le motif $\mu=b b 0 \epsilon_{1} \epsilon_{2}$.

Enfin, si le motif 11 est en n'importe quelle position, par invariance par translation, on obtient encore l'égalité des fonctions.

- Plus de deux états de 254 apparaissent. S'il n'apparaît qu'une suite de 1 , en combinant les deux cas précédents, on obtient l'égalité des fonctions. Le cas où $\mu=\eta_{1} \eta_{2} \epsilon \eta_{3} \eta_{4}$, donne toujours $\eta_{5} 3 \epsilon\left(\eta_{i} \in\{2,3\}\right)$.

Ainsi, les fonctions globales de 242 et de $114 \oplus_{240,1-1,1-1} 254$ agissent-elles de façon identique sur les configurations dans l'image de $\phi$.

\subsection{DÉCIDABILITÉ}

Les foulards permettent donc de mélanger deux règles de façon non triviale, qui n'est pas une simple superposition comme dans le cas des linéaires. On peut le voir encore sur la Figure 8 qui montre des orbites du mélange des règles 54 et 110 d'une part, de 54 et du décalage à droite d'autre part par le décalage à gauche.

Il ne s'agit pas non plus, contrairement à ce qui peut sembler lorsque l'on

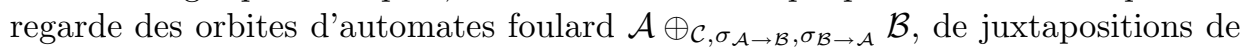
morceaux d'orbites des automates $\mathcal{A}$ et $\mathcal{B}$. Ceci est très visible sur la Figure 9. Il n'existe aucun motif carré $2 \times 2$ constitué de 1 dans aucun diagramme espacetemps de la règle 127, par définition même de la règle, alors que ce motif figure dans l'orbite de $127 \oplus_{170,0-1,0-1} 30$ de la figure. Cependant, il est possible, par une transformation géométrique convenable, de reconstituer une orbite de $\mathcal{A}$ ou $\mathcal{B}$ : c'est le cas de l'orbite $54 \oplus_{170,0-1,0-1} 240$ de la Figure 8 dont on peut extraire une orbite de 54 .

Il résulte de ces observations que même si $\mathcal{A}, \mathcal{B}$ et $\mathcal{C}$ ont des dynamiques décidables (en ce sens qu'il existe un programme qui, sur l'entrée $\langle i, j\rangle$, code d'une 

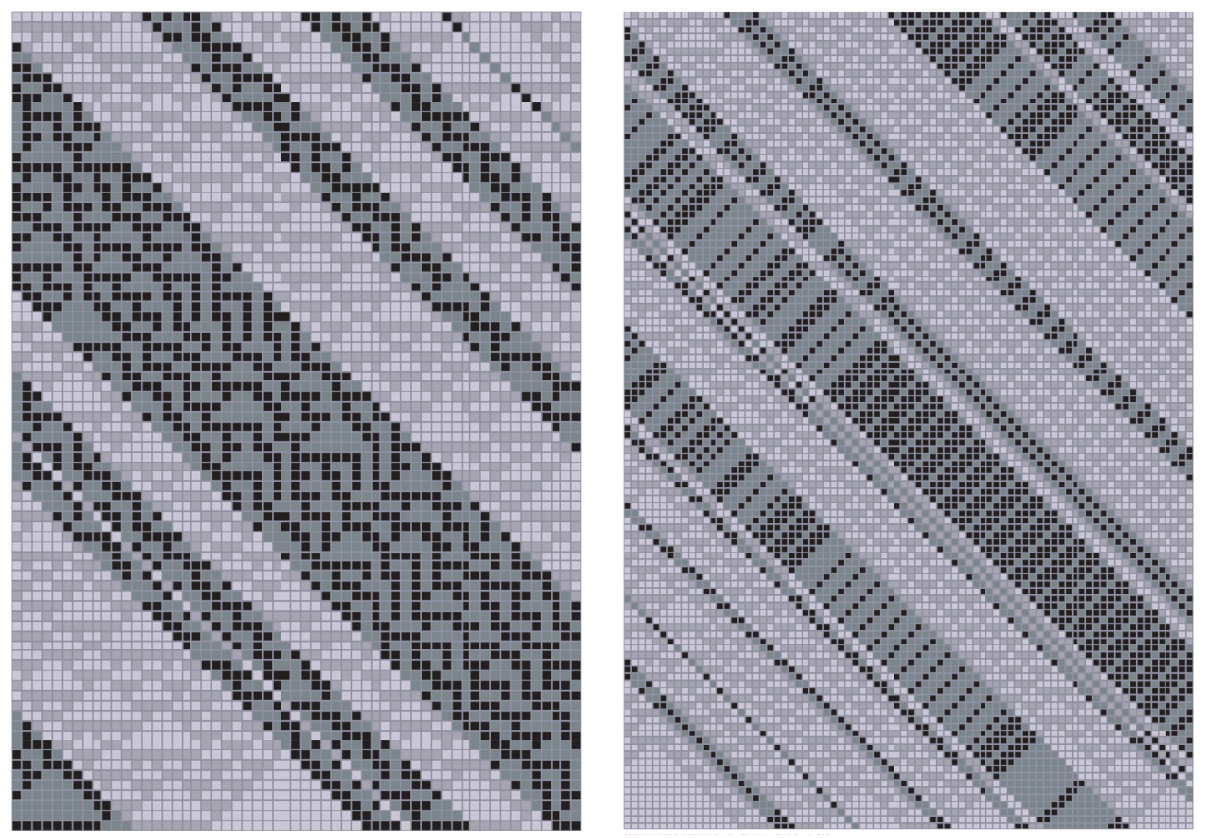

Figure 8. Exemple d'évolution des automates $54 \oplus_{170,0-1,0-1}$ 110 et $54 \oplus_{170,0-1,0-1} 240$ sur une ligne aléatoire de mesure markovienne.

configuration récursive et d'un motif de $\mathbb{Z}$, décide si le motif $j$ apparaît dans l'orbite de $i$ ), on conjecture que $\mathcal{A} \oplus_{\mathcal{C}, \sigma_{\mathcal{A} \rightarrow \mathcal{B}}, \sigma_{\mathcal{B} \rightarrow \mathcal{A}}} \mathcal{B}$ ne l'est pas en général. En effet, l'amalgame peut avoir comme conséquence de réintroduire des motifs ou des types de configurations (en particulier jardin d'Eden) qui n'apparaissent pas dans les évolutions des composantes. La dynamique des foulards n'est donc pas réductible à celle de ses composantes, et implique un véritable phénomène d'émergence.

\section{Conclusion}

L'auto-organisation est un phénomène beaucoup plus fréquent pour les automates captifs ou foulards qu'en général; en particulier, un choix approprié de paramètres permet d'obtenir presque à coup sûr de l'auto-organisation. Par ailleurs, l'embryon d'étude de ces classes montre qu'il serait pertinent de considérer un système dynamique agissant sur des parties finies ou infinies de $Q^{\mathbb{Z} \times \mathbb{N}}$ plutôt que sur les configurations de $Q^{\mathbb{Z}}$, fondé sur la notion de dépendance dont l'intérêt était déjà souligné par Grigorieff en 1985. En effet, la notion de jardin d'Eden n'est pas stable par composition. 

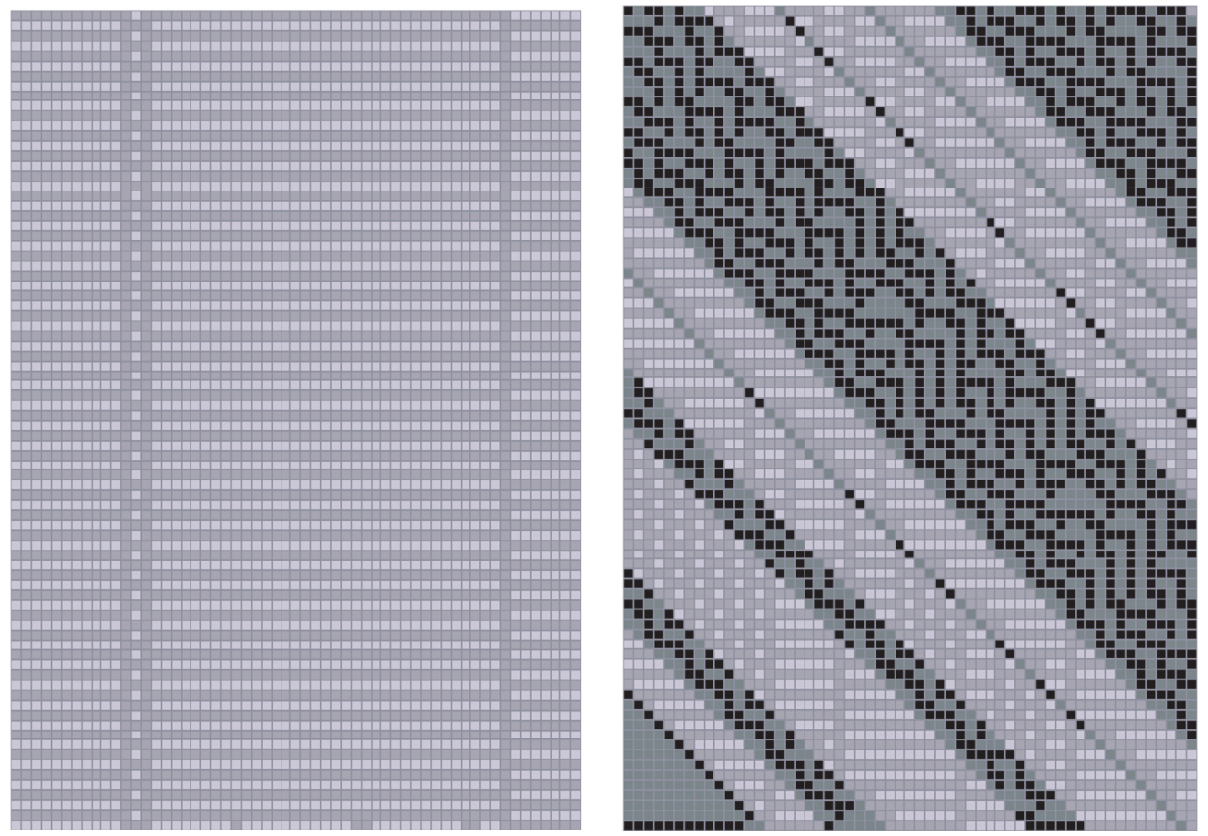

Figure 9. Exemple d'évolution de la règle 127 et de l'automate $127 \oplus_{170,0-1,0-1} 30$ sur une ligne aléatoire de mesure markovienne.

\section{RÉFÉRENCES}

[1] N. Boccara and M. Roger, Block transformations of one-dimensional deterministic cellulat automaton rules. J. Phys. A 24 (1991) 1849-1865.

[2] G. Cattaneo, E. Formenti, L. Margara and J. Mazoyer, Shift invariant distance on $s^{\mathbb{Z}}$ with non trivial topology, in Proceeding of MFCS'97, Springer Verlag (1997) 376-381.

[3] G. Cattaneo, E. Formenti, L. Margara and G. Mauri, Topological chaos and cellular automata. in Cellular Automata: a parallel model, edited by Delorme and Mazoyer, Springer Verlag (1999) 213-259.

[4] J.P. Crutchfield and J.E. Hanson, The attractor basin portait of a cellular automaton. J. Statist. Phys. 66 (1992) 1415-1462.

[5] J.P. Crutchfield and J.E. Hanson, Attractor vicinity decay for a cellular automaton. Chaos 3 (1993) 215-224.

[6] J.P. Crutchfield and J.E. Hanson, Turbulent pattern bases for a cellular automata. Phys. D 69 (1993) 279-301.

[7] J.P. Crutchfield and J.E. Hanson, Computational mechanics of cellular automata : an example. Phys. D 103 (1997) 169-189.

[8] K. Eloranta, Partially permutive cellular automata. Nonlinearity 6 (1993) 1009-1023.

[9] K. Eloranta, Random walks in cellular automata. Nonlinearity 6 (1993) 1025-1036.

[10] K. Eloranta, The dynamics of defect ensembles in one-dimensional cellular automata. $J$. Statist. Phys. 76 (1994) 1377-1398.

[11] K. Eloranta, Cellular automata for contours dynamics. Phys. D 89 (1995) 184-203.

[12] K. Eloranta and E. Nummelin, The kind of cellular automaton rule 18 performs a random walk. J. Statist. Phys. 69 (1992) 1131-1136. 
[13] P. Grassberger, Chaos and diffusion in deterministic cellular automata. Phys. D 10 (1984) 52-58.

[14] P. Grassberger, New mechanism for deterministic diffusion. Phys. Rev. A 28 (1984) 36663667.

[15] J.E. Hanson, Computational Mechnaics if Cellular Automata. Ph.D. Thesis, University of California, Ann Arbor, MI (1993). Published by University Microfilms.

[16] G. Hedlund, Endomorphism and automorphism of the shift dynamical system. Math. Syst. Theor. 3 (1969) 320-375.

[17] M. Hurley, Ergodic aspects of cellular automata. Ergod. Theor. Dyn. Syst. 10 (1990) 671685.

[18] M. Hurley, Varieties of periodic attractors in cellular automata. T. Am. Math. Soc. 326 (1991) 701-726.

[19] W. Hordijk, J.P. Crutchfield and M. Mitchell, Mechanisms of emergent computation in cellular automata. in Parallel Problem Solving in Nature V, edited by M. Schoenaur, A.E. Eiben, T. Bäck and K.-P. Schwefel. Lect. Notes Comput. Sci. (1998) 613-622.

[20] W. Hordijk, J.P. Crutchfield and C.R. Shalizi, Upper bound of the products of particle interactions in cellular automata. Phys. D 154 (2001) 240-258.

[21] P. Kůrka, Languages, equicontinuity and attractors in cellular automata. Ergod. Theor. Dyn. Syst. 17 (1997) 417-433.

[22] P. Kůrka, Cellular automata with vanishing particles. Fund. Inform. 58 (2003) 203-221.

[23] P. Kưrka, On the measure attractor of a cellular automaton. Discret. Contin. Dyn. Syst. (2005) S524-S535.

[24] P. Kưrka and A. Maass. Limit sets of cellular automata associated to probability measures. J. Statist. Phys. 100 (2000) 1031-1047.

[25] A. Maass, B. Host and S. Martinez, Uniform Bernoulli measure in dynamics of permutive cellular automata with algebraic local rules. Discrete Contin. Dyn. Syst. 9 (2003) 1423-1446.

[26] B. Martin, A group interpretation of particles generated by one-dimensional cellular automata. Int. J. Mod. Phys. C 11 (2000) 101-123.

[27] B. Martin, Automates cellulaires, information et chaos. Ph.D. Thesis, École Normale Supérieure de Lyon (2001).

[28] O. Martin, A. Odlysko and S. Wolfram, Algebraic properties of cellular automata. Commun. Math. Phys. 93 (1984) 219-258.

[29] J. Mazoyer and I. Rapaport, Inducing an order on cellular automata by a grouping operation, in Proceeding of STACS'98, Springer Verlag (1998) 128-227.

[30] H.V. McIntosh, A concordance for rule 110 (1999). http ://delta.cs.cinvestav.mx/mcintosh/comun

[31] H.V. McIntosh, Rule 110 as it relates to the presence of gliders (1999). http ://delta.cs.cinvestav.mx/mcintosh/comun/rule110.pdf

[32] H.V. McIntosh, Rule 110 is universal (1999). http ://delta.cs.cinvestav.mx/mcintosh/comun/texlet/texlet.pdf

[33] J. Nasser, N. Boccara and M. Roger, Particle-like structures and their interactions in spatiotemporal patterns generated ny one-dimentional cellular automata. Phys. Rev. A 44 (1991) $866-875$.

[34] N. Ollinger, Automates cellulaires : structures. Ph.D. Thesis, École Normale Supérieure de Lyon (2002).

[35] N. Ollinger, The quest for small universal cellular automata, in Proceeding of ICALP'02, 3 Springer Verlag (2002) 376-381.

[36] N. Ollinger, The intrinsic universality problem of one-dimensional cellular automata, in Proceeding of STACS'03, Springer Verlag (2003) 632-641.

[37] N. Ollinger and G. Richard, On the universality of rule 110, in Proceedings of DMTCS'O4 (2004).

[38] M. Pivato, Invariant measures for bipermutive cellular automata. Discret. Contin. Dyn. Syst. 12 (2005) 723-736. 
[39] M. Pivato, Algebraic invariants for crystallographics defects in cellular automata. Ergod. Theor. Dyn. Syst. 27 (2007) 199-240.

[40] M. Pivato, Defect particle kinematics in one-dimensional cellular automata. Theoret. Comput. Sci. 377 (2007) 205-225.

[41] M. Pivato, Spectral domain boundaries in cellular automata. Fund. Inform. 78 (2007) 417447.

[42] M. Mitchell, R. Das and J.P. Crutchfield, A genetic algorithm discovers particle-based computation in cellular automata, in Parallel Problem Solving in Nature III, edited by K.P. Schwefel, Y. Davidor and R. Männer. Lect. Notes Comput. Sci. (1994) 244-353.

[43] I. Rapaport, Ordre induit sur les automates cellulaires par l'opération de regroupement. Ph.D. Thesis, École Normale Supérieure de Lyon (1998).

[44] A. Smith, Real time languages by one-dimensional cellular automata. J. Comput. Syst. Sci. 6 (1972) 233-253.

[45] G. Theyssier, Captive cellular automata, in Proceeding of MFCS'04, Springer Verlag (2004) 427-438.

[46] G. Theyssier, Automates cellulaires : un modèle de complexité. Ph.D. Thesis, École Normale Supérieure de Lyon (2005).

[47] S. Wolfram, Theory and applications of cellular automata. World Scientific, Singapore (1986). 